Tropical Journal of Pharmaceutical Research April 2017; 16 (4): 877-883

ISSN: $1596-5996$ (print); 1596-9827 (electronic)

(C) Pharmacotherapy Group, Faculty of Pharmacy, University of Benin, Benin City, 300001 Nigeria.

All rights reserved.

Available online at http://www.tjpr.org

Original Research Article

http://dx.doi.org/10.4314/tjpr.v16i4.19

\title{
5,7-Dimethoxycoumarin inhibits neuronal apoptosis by targeting caspase- 3 expression in rats with acute cerebral infarction
}

\author{
Tao $\mathrm{Ma}^{1 \star}$ and Chunxia Wang ${ }^{2}$ \\ ${ }^{1}$ Department of Neurology, Xintai Municipal People's Hospital, Xintai 271200, ${ }^{2}$ Taishan Sanatorium of Shandong Province, \\ Taishan, Shandong 271000, China
}

*For correspondence: Email: taoma356@hotmail.com; Tel: 0086-0311-86556820

Received: 22 November 2016

Revised accepted: 16 March 2017

\begin{abstract}
Purpose: To investigate the effect of 5,7-dimethoxycoumarin on inhibition of neuronal apoptosis in a rat model of acute cerebral infarction.

Methods: Occlusion of middle cerebral artery was used for the preparation of a rat model of cerebral infarction. The rats in the treatment group were given $15 \mathrm{mg} / \mathrm{kg}$ dose of 5,7-dimethoxycoumarin daily for 30 days intraperitoneally. However, those in the normal control and model groups received the same volume of normal saline. The rats were sacrificed after anaesthetization to extract brain samples.

Results: Quantitative polymerase chain reaction $(P C R)$ analysis showed a significant $(p<0.02)$ decrease in the level of caspase-3 mRNA by 5,7-dimethoxycoumarin compared to the model group. Western blot assay data revealed significant decrease in caspase-3 protein expression by 5,7dimethoxycoumarin. The higher activity of caspase-3 in acute cerebral infarction rats was reduced significantly $(p<0.005)$ following treatment with $15 \mathrm{mg} / \mathrm{kg}$ dose of 5,7-dimethoxycoumarin for 30 days. Treatment of the acute cerebral infarction rats with $15 \mathrm{mg} / \mathrm{kg}$ dose of 5,7-dimethoxycoumarin daily for 30 days led to a greater decrease in apoptotic index than those of rats with model group $(p<0.005)$. 5,7-Dimethoxycoumarin treatment daily for 30 days at a dose of $15 \mathrm{mg} / \mathrm{kg}$ also caused a significant $(p<$ $0.005)$ decrease in neurological impairment score compared to the model animal group.

Conclusion: 5,7-Dimethoxycoumarin successfully inhibits neuronal apoptosis via inhibition of the expression of caspase-3 in brain tissues and thus may be useful for cerebral infarction therapy.
\end{abstract}

Keywords: Cerebral infarction, 5,7-Dimethoxycoumarin, Neurological impairment, Apoptosis, Caspase3

Tropical Journal of Pharmaceutical Research is indexed by Science Citation Index (SciSearch), Scopus, International Pharmaceutical Abstract, Chemical Abstracts, Embase, Index Copernicus, EBSCO, African Index Medicus, JournalSeek, Journal Citation Reports/Science Edition, Directory of Open Access Journals (DOAJ), African Journal Online, Bioline International, Open-J-Gate and Pharmacy Abstracts

\section{INTRODUCTION}

Acute cerebral infarction is a cerebrovascular disease and has very high incidence and mortality rate throughout the world [1,2]. The disease is characterized by blockage of blood to brain tissues leading to ischemia and hypoxia followed by necrosis of cerebral tissues [3]. The advanced stage of the disease involves generation of reactive oxygen radicals inducing neuronal cell death and subsequent activity impairment $[4,5]$. It is reported that during cerebral infarction in animals the rate of neuronal apoptosis is very high [6]. One of the important factors expressed in higher level and associated with the induction of neuronal apoptosis is the caspases [7-9]. In the brain tissue samples of cerebral infarction patients the expression of caspase-3 has been found to be markedly higher [10]. Thus inhibition of caspase- 3 expression in 
patients with cerebral infarction can play a vital role in the treatment of the disease. Therefore, various studies have been performed to investigate the effect of anti-oxidants on the pathology of cerebral infarction [11].

Coumarins, isolated in abundance from plant source exhibit promising anti-oxidant property $[12,13]$. Both natural as well as synthetic coumarins are in clinical trials for the treatment of various types of cancers because of their tendency to induce carcinoma cell apoptosis [14]. 5,7-Dimethoxycoumarin (Figure 1), a derivative of coumarin present in vegetables has shown potent anti-cancer activity through induction of DNA damage and inhibition of carcinoma cell proliferation $[15,16]$. The chemical structure of 5,7-dimethoxycoumarinindicates that it has potential to act as anti-oxidant agent. 5,7dimethoxycoumarin also causes cell cycle arrest in melanoma cell lines resulting in inhibition of cell proliferation [17].The current study was designed and performed to investigate the effect of 5,7-dimethoxycoumarin on inhibition of apoptosis induction in the rat model of acute cerebral infarction. The study demonstrated that 5,7-dimethoxycoumarin successfully inhibited neuronal apoptosis through targeting the expression of caspase-3 in brain tissues.<smiles>COc1cc(OC)c2ccc(=O)oc2c1</smiles>

Figure 1: Chemical structure of 5,7dimethoxycoumarin

\section{EXPERIMENTAL}

\section{Animals and ethical approval}

38-weeks old male Sprague-Dawley rats ( 210 g) used in the study were purchased using Animal licence: SCXK (no. Hebei 2014/213/41/56) from the Experimental Animal Center of Hebei Medical University. Fifteen days before the start of actual experiment, the animals were acclimatized to the laboratory environment. All the rats were housed under $12 \mathrm{~h}$ light/dark cycle in groups of $2 /$ cage. The room was maintained at a humidity of $50-60 \%$ and temperature of $23 \pm 1{ }^{\circ} \mathrm{C}$. All the animals had free access to food and water. Approval for the study was taken from the Ethics Committee of the Xintai Municipal People's Hospital (approval no. CK43140407). All experimental animal procedures were conducted in compliance with State and Federal laws, standards of the US Department of Health and Human Services, and guidelines established by Tulane University Animal Care and Use Committee, accredited by Association for the Assessment and Accreditation of Laboratory Animal Care [18]. Efforts were made to reduce animal suffering to the minimum possible.

\section{Preparation of animal model of acute cerebral infarction}

For the preparation of acute cerebral infarction, a rat model cerebral artery infarction method reported earlier was used [19]. The establishment of animal model was confirmed by carefully examining the rats after surgery. The rats were then divided into three groups of 10 each; normal control, model and the treatment groups. The rats in the treatment group were injected $15 \mathrm{mg} / \mathrm{kg}$ doses of 5,7dimethoxycoumarin daily for 30 days whereas those in the model and normal control group received the same volume of normal saline.

\section{RT-PCR analysis}

Total RNA from the brain tissue samples was isolated using TRIzol (Invitrogen, Carlsbad, CA, USA) as per the instructions mentioned in the manual protocol. The extracted RNA (1 $\mu \mathrm{g})$ samples were subjected to reverse transcription using M-MLV reverse transcriptase and the Random-pdN6 Primer (Invitrogen). Amplification of the signals was performed using polymerase chain reaction (PCR) as follows: in the $50 \mu \mathrm{L}$ water was taken template cDNA $(2 \mu \mathrm{L})$, dNTPs (0.2 mM), $1.25 \mathrm{U} / \mu \mathrm{L}$ Go Taq polymerase (Promega, Madison, WI, USA) along with the various primers (0.5 $\mu \mathrm{M}$ each). It was then denatured by heating at $95{ }^{\circ} \mathrm{C}$ for $30 \mathrm{~s}$ and subsequently annealed for $30 \mathrm{sec}$ at melting temperature. Elongation over 30 cycles was performed at $72{ }^{\circ} \mathrm{C}$ for 1 -min. The sequence of used caspase-3 primer was: forward, 5'-GGT ATT GAG ACA GAC AGT GG-3' and reverse, 5'CAT GGG ATC TGT TTC TTT GC-3'. Electrophoresis of the PCR products was performed on $2.0 \%$ agarose gel and photographed under UV.

\section{Western blot analysis}

The brain tumor samples from the rats were collected, washed with cold PBS and subsequently treated with lysis buffer. After treatment with ice-cold lysis buffer for $45 \mathrm{~min}$, the lysates were subjected to centrifugation for 15 $\min$ at $4{ }^{\circ} \mathrm{C}$ at $12,000 \times \mathrm{g}$. The supernatant was 
then collected and the concentration of proteins in the samples was analysed using Bicinchoninic Acid Protein Assay kit (Pierce, Rockford, IL, USA). The $50 \mu \mathrm{g}$ protein samples were subjected to electrophoresis over $12 \%$ sodium dodecyl sulphate-polyacrylamide gel. The protein samples were transferred to polyvinylidene difluoride (PVDF) membranes using a semidry transfer system (Bio-Rad Laboratories, Hercules, CA, USA). The non-specific sites in the membranes were incubated with $5 \%$ bovine serum albumin and Tris-buffered saline with Tween® 20 buffer for $3 \mathrm{~h}$. The bands were incubated with a specific rabbit anti-human antibody against caspase-3 mRNA (Cell Signaling Technology, Beverly, MA, USA). The bands were normalized to $\beta$-actin concentration. The complex development was performed using an ECL detection kit as per the manufacturer's instructions (ECL GST western blotting detection kit, Pierce Biotechnology, Inc., Waltham, MA, USA). For the purpose of imaging, GelGDoc2000 imaging system (Bio-Rad Laboratories $\mathrm{GmbH}$, Munich, Germany) was employed.

\section{Examination of neurological impairment}

Analysis of the impairment in neurological activity was performed using the known scale developed by Bederson [20]. The score of neurological impairment after surgery on the scale was assigned as follows: No neurological impairment symptoms $=0$ score; problem in front paw extension = 1 score; rolling of body to left side = 2 ; tilting to one side only $=3$ and complete movement loss $=4$.

\section{Analysis of caspase- 3 activity}

The activity of caspase-3 was analysed using commercially available reagent for detection of caspase-3 protein action (Beyotime Institute of Biotechnology, Beijing, China).

\section{Terminal deoxynucleotidyl transferase dUTP nick end labeling (TUNEL) assay}

Brain tissue sample apoptosis was analysed using the In Situ Cell Death Detection kit, POD (Roche). The brain tissue samples were embedded in paraffin after washing with PBS and cut into thin $2 \mu \mathrm{m}$ sections. The sections were then de-parrafined in boiling xylene and treated with hydrogen peroxide. The sections were fixed with $4 \%$ paraformaldehyde and then subjected to counter staining for $10 \mathrm{~min}$ with 4',6diamidino-2-phenylindole under dark atmosphere. The sections were observed carefully for apoptotic cells (TUNEL-positive cells) under a fluorescence microscope
(Olympus IX70; Olympus Corporation, Tokyo, Japan).

\section{Statistical analysis}

SPSS software, version 17.0 (SPSS, Inc., Chicago, IL, USA) was employed for the analysis of the data. The data are presented as mean \pm standard deviation $(n=3)$. The variance analysis was used for the comparison of the data among the groups. The differences were taken as statistically significant at $p<0.05$.

\section{RESULTS}

\section{Effect of 5,7-dimethoxycoumarin on caspase- 3 mRNA levels}

Quantitative PCR analysis showed a significantly $(p<0.05)$ higher level of caspase-3 mRNA in the rats with acute cerebral infarction compared to the normal control group (Figure 2). Treatment of the rats with 5,7-dimethoxycoumarin caused a significant $(p<0.02)$ reduction in the level of caspase-3 mRNA than those in the model group (Figure 2). Analysis of the caspase-3 protein level in rats of the model group revealed significantly higher level than those of the normal control rats (Figure 2). 5,7-Dimethoxycoumarin treatment of the rats with acute cerebral infarction caused a significant decrease in the level of caspase-3 protein compared to the model animal group (Figure 2). The level of both mRNA and protein corresponding to caspase-3 was thus reduced in the rats with acute cerebral infarction on treatment with $15 \mathrm{mg} / \mathrm{kg}$ dose of 5,7-dimethoxycoumarin.

\section{Effect of 5,7-dimethoxycoumarin on caspase- 3 activity}

Analysis of the activity of caspase- 3 in the rats with acute cerebral infarction revealed significantly $(p<0.05)$ higher level than those of rats in the normal control group. The higher activity of caspase- 3 in acute cerebral infarction rats was reduced significantly on treatment with $15 \mathrm{mg} / \mathrm{kg}$ dose of 5,7-dimethoxycoumarin for 30 days. Caspase- 3 activity in the normal, control and treatment groups was 2, 15 and 3.6, respectively. On the day $30^{\text {th }}$ of treatment, level of caspase- 3 activity in the normal control and 5,7-dimethoxycoumarin treatment groups was found to be similar.

\section{Effect of 5,7-dimethoxycoumarin on apoptotic index and infarction scope}

The apoptotic index in rats with acute cerebral infarction was markedly higher compared to 

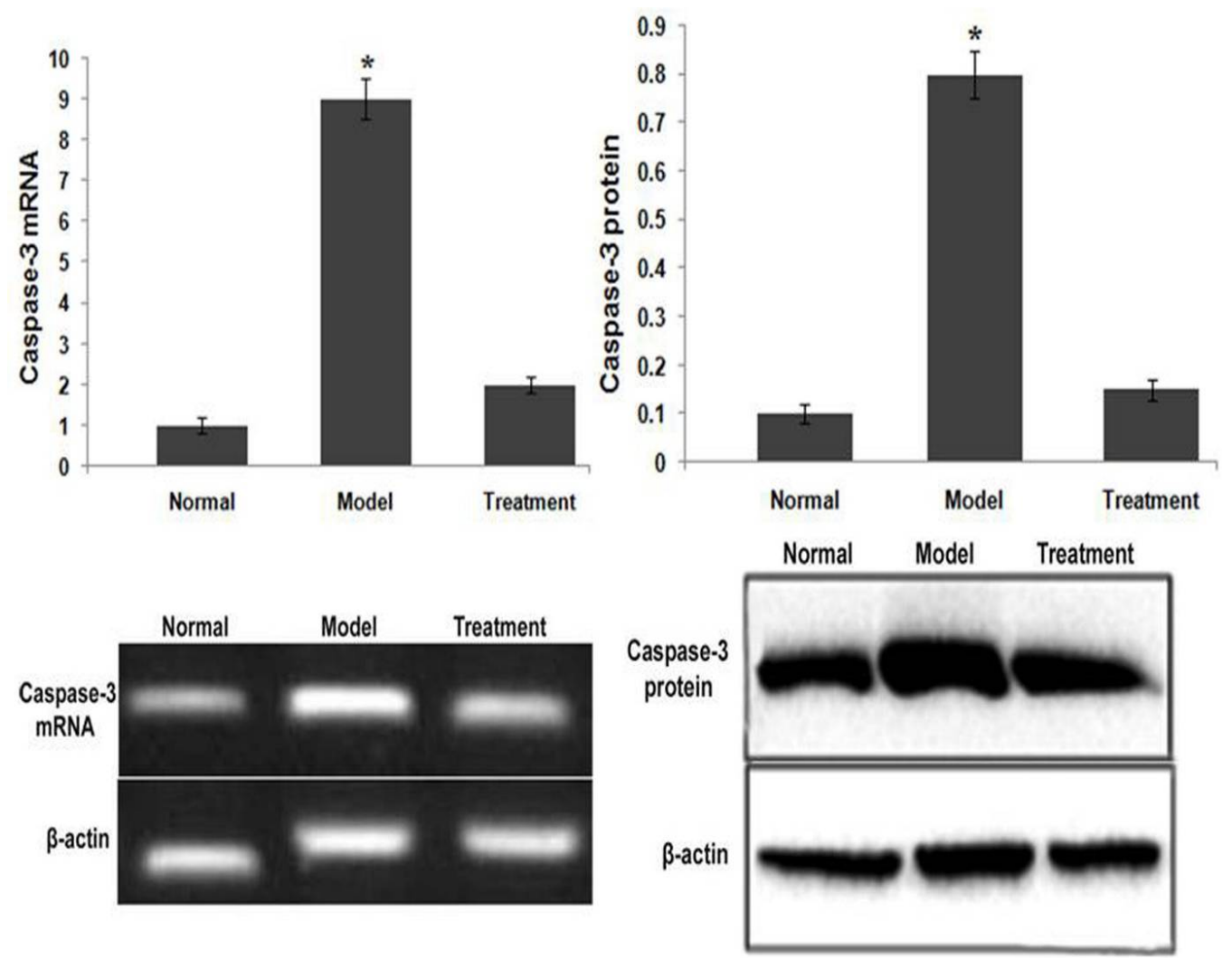

Figure 2: 5,7-dimethoxycoumarinprevents increase in expression level of mRNA and protein corresponding to caspase- 3 in the rats with acute cerebral infarction. Rats with acute cerebral infarction were treated for 30 days with $15 \mathrm{mg} / \mathrm{kg}$ dose of 5,7-dimethoxycoumarin daily. The expression level of caspase-3 mRNA and proteins in the rat brain samples was analysed using qPCR and western blot assay, respectively

normal control (Figure 3). However, treatment of the acute cerebral infarction rats with $15 \mathrm{mg} / \mathrm{kg}$ dose of 5,7-dimethoxycoumarin daily for 30 days led to a significant decrease in apoptotic index than those of rats in the model group (Figure 3).

\section{Effect of 5,7-dimethoxycoumarin on neurological impairment in rats with acute cerebral infarction}

In the rats with acute cerebral infarction, neurological impairment was found to be markedly higher compared to the normal control group (Figure 4). 5,7-dimethoxycoumarin treatment daily for 30 days at a dose of $15 \mathrm{mg} / \mathrm{kg}$ caused a significant decrease in the neurological impairment score compared to the model animal group.

\section{DISCUSSION}

Coumarins, natural phytochemical products obtained from plants possess promising antioxidant activity [12,13]. Many coumarin molecules are in clinical trials against various types of diseases including carcinoma [14]. Apoptosis, programmed death of cells is induced through the generation of reactive oxygen species in cells which damage DNA and affect cellular pathways. In brain tissue samples of rats and patients the rate of neuronal apoptosis has been found to be markedly high [21]. Therefore, in the current study effect of the anti-oxidant, 5,7dimethoxycoumarin on prevention of apoptosis induction in neuronal cells of acute cerebral infarction rats was investigated. The main factor responsible for induction of cell apoptosis is a protease, caspase-3 which triggers cellular pathways involved in neuronal apoptosis [22]. In the current study, the expression of both mRNA and protein corresponding to caspase- 3 in the brain samples of rats with acute cerebral infarction was markedly higher. However, 5,7dimethoxycoumarin prevented the increase in the level of mRNA and protein corresponding to caspase- 3 in the rats.

The higher activity of caspase-3 in acute cerebral infarction rats was reduced significantly on treatment with 5,7-dimethoxycoumarin for 30 days. Caspase-3 is considered to be the significant target for prevention of acute cerebral infarction because of its major role in ischemia and induction of apoptosis [23,24]. In the current 


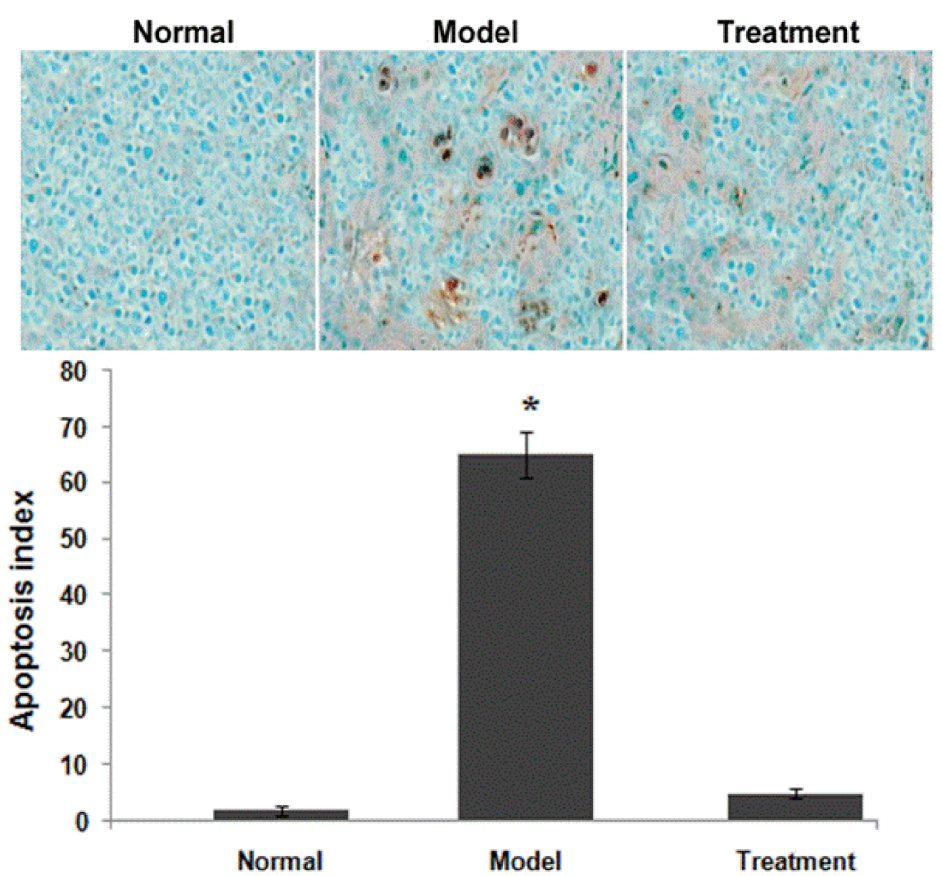

Figure 3: 5,7-Dimethoxycoumarin prevents increase in apoptosis index in the rats with acute cerebral infarction. The brain samples of rats with acute cerebral infarction after treatment with 5,7-dimethoxycoumarin were analysed for apoptotic index using TUNEL staining. Quantification of neuronal cell apoptosis was performed using TUNEL staining

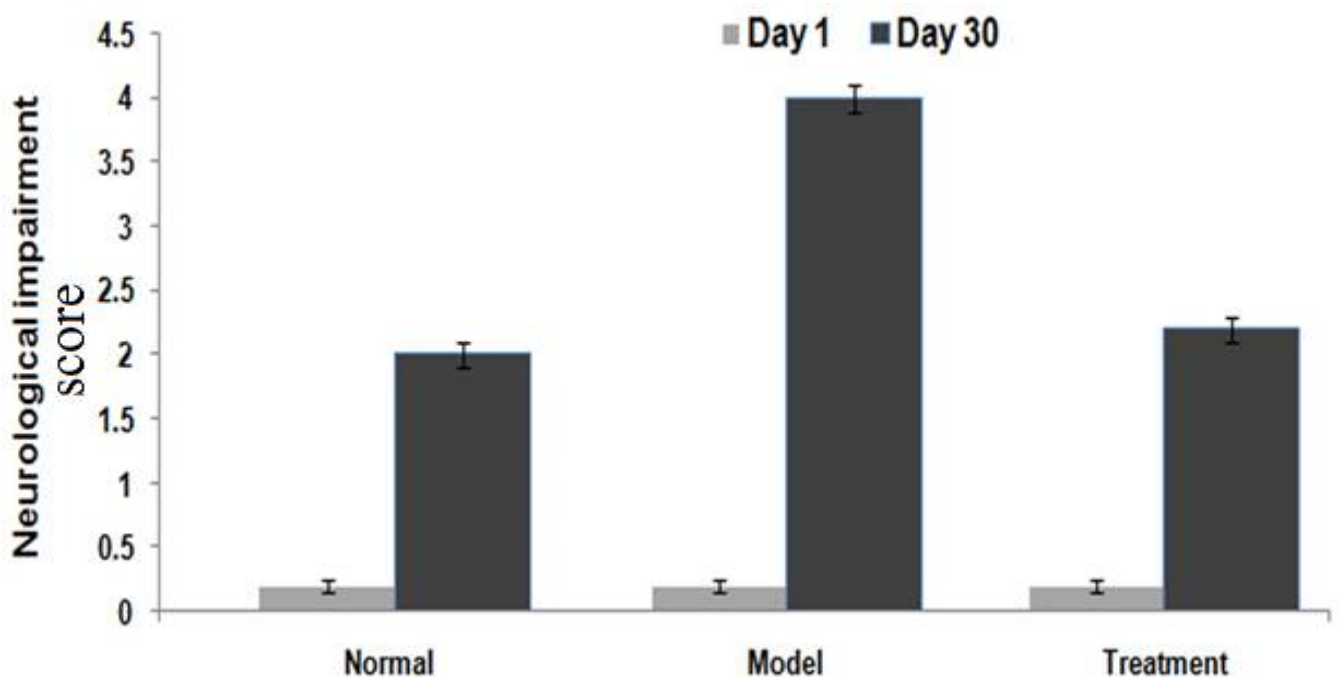

Figure 4: 5,7-Dimethoxycoumarin prevents the increase in neurological impairment score in rats with acute cerebral infarction. The rats in the treatment group were injected with $15 \mathrm{mg} / \mathrm{kg}$ dose of 5,7-dimethoxycoumarin daily for 30 days and then neurological impairment score was examined.

study, the apoptotic index of rats with acute cerebral infarction was markedly higher than for normal control. However, treatment of the acute cerebral infarction rats with 5,7dimethoxycoumarin daily for 30 days led to a significant decrease in the apoptotic index than those of rats in the model group. In the rats with acute cerebral infarction, neurological impairment was markedly higher compared to the normal control group. 5,7-Dimethoxycoumarin treatment daily for 30 days caused a significant decrease in the neurological impairment score compared to the model animal group.

\section{CONCLUSION}

The findings of the present study demonstrate that 5,7-dimethoxycoumarin successfully inhibits neuronal apoptosis by inhibition of expression of caspase-3 in brain tissues. Therefore, 5,7dimethoxycoumarin can potentially be used for the treatment of acute cerebral infarction in patients. 


\section{DECLARATIONS}

\section{Acknowledgement}

The authors thank Xintai Municipal People's Hospital for giving a grant for this study and also for making available the required facilities.

\section{Conflict of Interest}

No conflict of interest associated with this work.

\section{Contribution of Authors}

The authors declare that this work was done by the authors named in this article and all liabilities pertaining to claims relating to the content of this article will be borne by them.

\section{Open Access}

This is an Open Access article that uses a funding model which does not charge readers or their institutions for access and distributed under the terms of the Creative Commons Attribution License (http://creativecommons.org/licenses/by/ 4.0) and the Budapest Open Access Initiative (http://www.budapestopenaccessinitiative.org/rea d), which permit unrestricted use, distribution, and reproduction in any medium, provided the original work is properly credited.

\section{REFERENCES}

1. Arboix A, Alio J. Acute cardioembolic cerebral infarction: answers to clinical questions. Curr Cardiol Rev 2012, 8: 54-67.

2. Mokin M, Snyder KV, Siddiqui AH, Hopkins LN, Levy El. Endovascular management and treatment of acute ischemic stroke. Neurosurg Clin N Am 2014; 25: 583592.

3. Hsieh Fl, Chiou HY. Stroke: morbidity, risk factors and care in Taiwan. J Stroke 2014; 16: 59-64.

4. Candelario-Jalil E. Injury and repair mechanisms in ischemic stroke: considerations for the development of novel neurotherapeutics. Curr Opin Investig Drugs 2009; 10: 644-654.

5. Sergeeva SP, Litvickiy PF, Gultyaev MM, Savin AA, Breslavich ID. To the Fas-induced neurons apoptosis mechanisms in stroke pathogenesis. Patol Fiziol Eksp Ter 2013; 3: 15-18 (In Russian).

6. Wang JP, Yang ZT, Liu C, He YH, Zhao SS. L-carnosine inhibits neuronal cell apoptosis through signal transducer and activator of transcription 3 signaling pathway after acute focal cerebral ischemia. Brain Res 2013; 1507: 125-133.
7. Fan W, Dai Y, Xu H. Caspase-3 modulates regenerative response after stroke. Stem Cells 2014; 32: 473-486.

8. Yang $B$, Ye D, Wang $Y$. Caspase-3 as a therapeutic target for heart failure. Expert Opin Ther Targets 2013; 17: 255-263.

9. Zhang ZN, Li JY, Zhao Y, Wang JQ, Huang C, Fan GQ. Effects of Tongnao Huoluo acupuncture therapy on Caspase-3 and Bcl-2 of rats with acute cerebral infarction. Zhongguo Zhong Xi Yi Jie He Za Zhi 2013; 33: 646-650 (In Chinese).

10. Rosell A, Cuadrado E, Alvarez-Sabín J. Caspase-3 is related to infarct growth after human ischemic stroke. Neurosci Lett 2008; 430: 1-6.

11. Kikuchi K, Tanaka E, Murai Y, Tancharoen S. Clinical trials in acute ischemic stroke. CNS Drugs 2014; 28 : 929-938.

12. Egan D, O'Kennedy R, Moran E, Cox D, Prosser E, Thornes RD. The pharmacology, metabolism, analysis, and applications of coumarin and coumarin-related compounds. Drug Metab Rev 1990; 22: 503-529.

13. Maucher A, Kager M, von Angerer E. Evaluation of the antitumour activity of coumarin in prostate cancer models. J Cancer Res Clin Oncol 1993; 119: 150-154.

14. Lake BG. Coumarin metabolism, toxicity and carcinogenicity: relevance for human risk assessment. Food Chem Toxicol 1999; 37: 423-453.

15. Salvatore A, Borkosky S, Willink E, Bardòn A. Toxic effects of lemon peel constituents on Ceratitis capitata. $J$ Chem Ecol 2004; 30: 323-333.

16. Kawaii S, Tomono Y, Katase E, Ogawa K, Yano $M$. Isolation of furocoumarins from bergamot fruits as $H L-60$ differentiation-inducing compounds. J Agric Food Chem 1999; 47: 4073-4078.

17. Alesiani D, Cicconi R, Mattei M, Montesano C, Bei R, Canini $A$. Cell cycle arrest and differentiation induction by 5,7dimethoxycoumarin on melanoma cell lines. Int $\mathrm{J}$ Oncol 2008; 32: 425-434.

18. Jeong EK, Lee SY, Jeon HM. Role of extracellular signal regulated kinase (ERK)1/2 in multicellular resistance to docetaxel in MCF-7 cells. Int J Oncol 2010; 37: 655-661.

19. Belayev L, Alonso OF, Busto R, Zhao W, Ginsberg MD. Middle cerebral artery occlusion in the rat by intraluminal suture. Neurological and pathological evaluation of an improved model. Stroke 1996; 27: 1616-1622.

20. Longa EZ, Weinstein PR, Carlson S, Cummins $R$. Reversible middle cerebral artery occlusion without craniectomy in rats. Stroke 1989; 20: 84-91.

21. Ouyang L, Shi Z, Zhao S, Wang FT, Zhou TT, Liu B, Bao JK. Programmed cell death pathways in cancer: $A$ review of apoptosis, autophagy and programmed necrosis. Cell Prolif 2012; 45: 487-498.

22. Charriaut-Marlangue C. Apoptosis: A target for neuroprotection. Therapie 2004; 59: 185-190.

23. Yang B, Ye D, Wang Y. Caspase-3 as a therapeutic target for heart failure. Expert Opin Ther Targets 2013; 17: 255-263.

24. Ferrer I, Friguls B, Dalfó E, Justicia C, Planas AM. Caspase-dependent and caspase-independent

Trop J Pharm Res, April 2017; 16(4): 882 
Ma \& Wang

signalling of apoptosis in the penumbra following middle cerebral artery occlusion in the adult rat. Neuropathol
Appl Neurobiol 2003; 29: 472-481. 